\title{
Crystallization in a horizontally vibrated monolayer of spheres
}

\author{
G. Straßburger and I. Rehberg* \\ Institut für Experimentelle Physik, Otto-von-Guericke-Universität Magdeburg, Postfach 4120, D-39016 Magdeburg, Germany
}

(Received 7 March 2000)

\begin{abstract}
The dynamics of a cluster consisting of a few thousand steel spheres on a horizontally vibrated plate is studied experimentally. A transition from randomly arranged and almost independently moving particles to a two-dimensional crystallike structure occurs when the filling fraction (the number of particles) is increased. This transition is quantitatively characterized by an order parameter obtained from the pair distribution function of the spheres.
\end{abstract}

PACS number(s): 45.05.+x, 64.60.Cn, 81.05.Rm, 83.10.Pp

Granular matter like sand, cosmic dust, and popcorn consists of a large number of grains, and it is thus tempting to describe it in terms of models adapted from the kinetic theory of gases. However, the interaction between the particles is dissipative, which makes granular matter dissimilar to systems treated by equilibrium thermodynamics: the granulate must be continuously supplied with energy to keep the grains moving. Still, such a state, in which the grains are kept in disordered motion, is called gaslike, and the term granular gas is frequently used. If the energy flux into the system is reduced, the gaslike state may decay into a more ordered one. Transitions between such different states are referred to as granular phase transitions [1]. Their importance has been stressed in connection with the coagulation of planetesimals into planets [2]. Moreover, the study of such transitions provides quantitative information about collective dissipation mechanisms in the granulate.

In principal, granular phase transitions can be induced by changing the amount of mechanical energy flowing into the granulate, or the physical properties of the granulate. Particularly convincing experimental examples are documented in Refs. [3] and [4], where coexistence of a solidlike and a fluidlike state is nicely demonstrated for vertically vibrated monolayers of spheres. The solid state is characterized by contact between the vibrating plate and the particle, while in the gaslike state the particles loose contact for some time interval within the oscillation period. Thus, the phase transition coincides with the onset of particle motion in the vibrating system. This is also the case for a horizontally vibrated vertical layer of spheres. Here it is possible to pinpoint a phase transition that coincides with the onset of particle motion in a comoving frame [5].

The situation is fairly different for a horizontal monolayer of spheres under horizontal vibration, which is studied in this paper. Here the spheres do move even for very small values of the acceleration of the container, provided it is filled with less than one complete monolayer. We find a phase transition between two different fluidized phases: For low-packing densities of the granulate, we observe a gaslike phase, and at higher densities a fluidized phase with crystallike order. We

\footnotetext{
*Present address: Universität Bayreuth, Physikalisches Institut, D-95440 Bayreuth, Germany.
}

pinpoint this transition from a granular gas into a dynamic crystallike state by an order parameter extracted from the pair distribution function.

The experimental setup consists of different numbers (500-5000) of steel spheres (diameter $d=1 \mathrm{~mm}$ ) placed in a flat container $(21.5 \mathrm{~cm} \times 14.5 \mathrm{~cm} \times 2 \mathrm{~cm})$ made of aluminum. Horizontal sinusoidal vibration of the container at a constant driving frequency of $4 \mathrm{~Hz}$ feeds the granulate with energy. The driving amplitudes were $1 \mathrm{~cm}$ and $2 \mathrm{~cm}$, which corresponds to dimensionless accelerations $\left(\Gamma=\omega^{2} A / g\right)$ of 0.64 and 1.29. The vibration apparatus is the same as described in Ref. [5]. An aging of the spheres by work hardening the surfaces of the spheres turned out to be necessary for getting reproducible results. A charge-coupled-device (CCD) camera connected to a frame grabber is used for detecting the spheres.

Figure 1 shows snapshots of the particle arrangement in the central part of the container $(6.65 \mathrm{~cm} \times 6.65 \mathrm{~cm})$ for

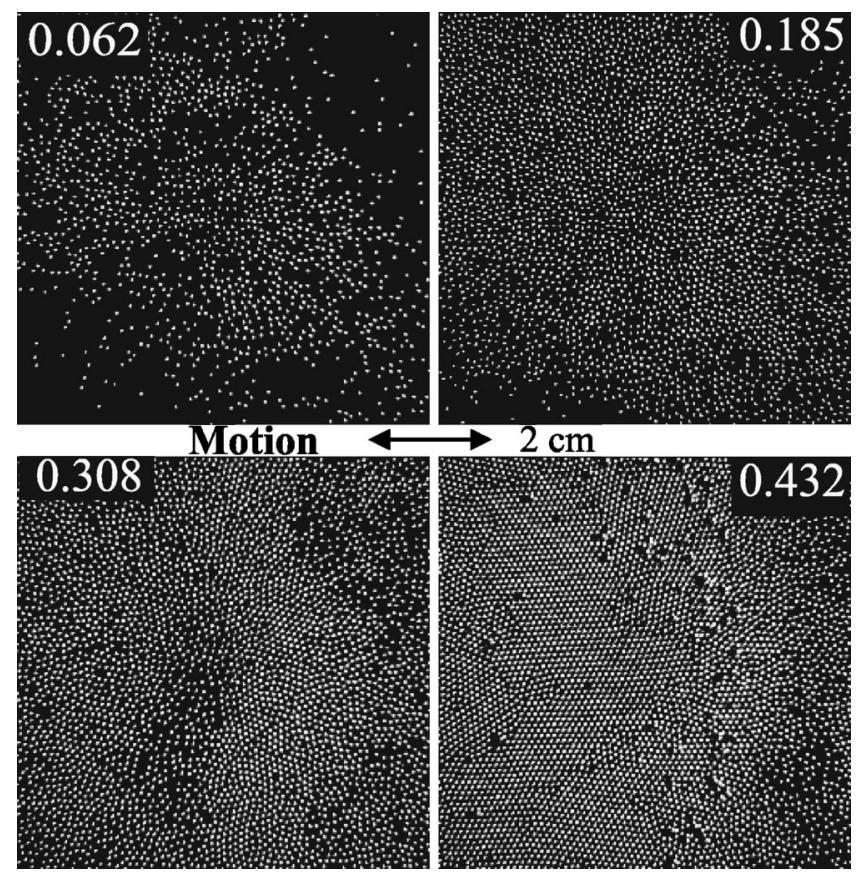

FIG. 1. Snapshots of the central area of the container taken at the phase of maximum acceleration. The numbers indicate the filling fraction. The arrow indicates the horizontal motion of the container. 


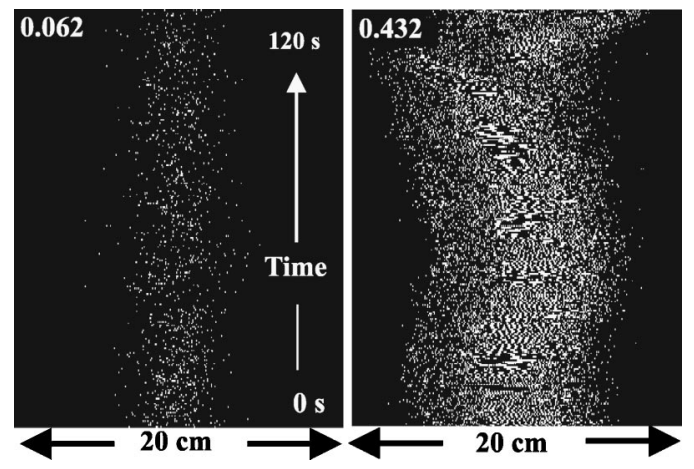

FIG. 2. 480 consecutive lines are plotted on top of each other, giving the temporal behavior of the granulate over a time interval of $120 \mathrm{~s}$.

four different filling fractions $\nu$. The oscillation amplitude is $1 \mathrm{~cm}$. The snapshots are taken at a phase of maximal acceleration, i.e., minimal velocity, of the container. The upperleft image is taken with a mass of $10 \mathrm{~g}$ granulate in the container, which corresponds to about $2450 \pm 20$ particles. The area covered by spheres divided by the area of the container is then $\nu=0.062$. This global filling fraction $\nu$ is smaller than the (local) filling fraction one would estimate from the inspection of the image. This discrepancy can be observed for all images presented here. It is due to the influence of the container walls, which reflect and accelerate the particles, thus pushing them into the middle of the container. This effect has most clearly been demonstrated to occur in situations where the "heating" of the granulate is exclusively due to the side walls, both in experiments [6] and numerical simulations (see Fig. 2 of Ref. [7]).

The upper-right image of Fig. 1 is taken with about 7350 particles corresponding to $\nu=0.185$. Here the clusterlike regime of higher density of particles fills almost the entire image. At $\nu=0.308$ the cluster seems to show some structure, namely, regimes of different particle density. For a filling fraction of $\nu=0.432$, the snapshot shows a crystallike structure. This structure is reminiscent of the ordered state obtained in Ref. [3] in the dense system. Before characterizing the transition from the gaslike to the crystallike structure quantitatively, we focus on qualitative aspects like the spatio-temporal behavior of the granulate for two of the filling fractions used in Fig. 1. This is shown in Fig. 2. One single line of length $20 \mathrm{~cm}$ is detected every $40 \mathrm{~ms}$, and the measured intensities are plotted on top of each other. The white dots represent particles, which clearly concentrate around the middle of the container-both for $\nu=0.062$ and $\nu=0.432$ - as a result of the additional "heating" caused by the side walls of the container. The gas-crystal transition occurs for moving particles, rather than being accompanied with a transition from a motionless ground state. The righthand side of Fig. 2 also indicates a slow motion of the crystallike cluster, an interesting feature that has not yet been analyzed in any detail.

This transition scenario changes when a higher driving amplitude of $2 \mathrm{~cm}$ (see Fig. 3) is used. At $\nu=0.062$ it becomes apparent that the mean distance between the particles is higher than in the corresponding part of Fig. 1. Obviously, the tendency for cluster formation is reduced because of the higher energy input provided at the larger driving amplitude.

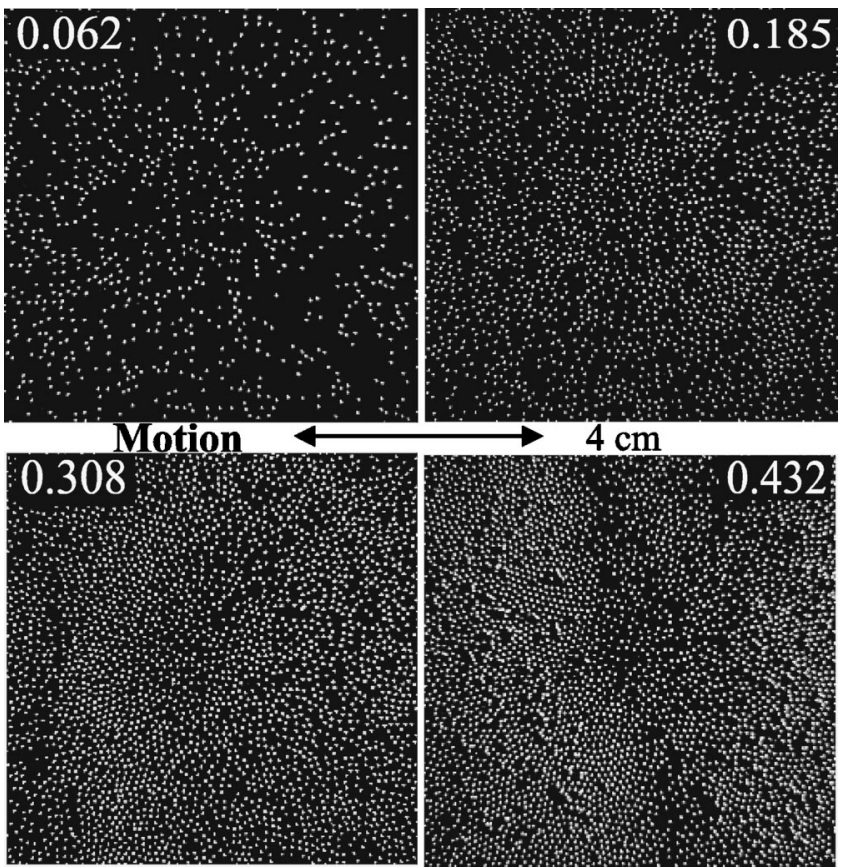

FIG. 3. Snapshots obtained at a driving amplitude of $2 \mathrm{~cm}$.

This tendency can also be observed at $\nu=0.185$ and $\nu$ $=0.308$. In the lower-right part of Fig. 3 a new scenario appears: Some particles loose contact to the bottom of the container, i.e., the arrangement of the particles becomes three dimensional. The formation of a regular crystal like arrangement is thus not observable in this case, which means that the driving amplitude of $2 \mathrm{~cm}$ is too large for the twodimensional granular phase transition considered here. In the central part one sees a dark zone with lower density of particles that divides two regions of higher density of particles. This is indicative for the trend to form a regular array of three-dimensional clusters, which can be observed in longer containers, and have been described in Refs. [8] and [9].

In order to characterize the transition from gaslike to crystallike behavior of the cluster, we increased the number particles step-by-step (step size $1 \mathrm{~g}$, corresponding to 245 particles) at constant dimensionless acceleration amplitudes. We took images during the phase of minimal velocity of the driving motion (as in Figs. 1 and 2). By subsequent image processing we determine the coordinates of the center of gravity of each sphere.

A suitable order parameter for quantifying the granular phase transition is obtained from a slightly modified pair distribution function. To obtain it, we calculate the distances of all pairs of grains. These separations are sorted into a histogram $h(r)$. To account for finite-size effects of our observation area, which would lead to an underestimation of large separations, we use a normalization histogram $n(r)$. $n(r)$ is the histogram of separations for a $1024 \times 1024$ square matrix of particles. This matrix reflects the resolution of the commercial image processing algorithm for the determination of the particle centers, which was obtained after zooming the pixel grid of the CCD cameras with a factor of 4 . We normalize the histogram via $n_{0}(r)=n(r) A / M^{2}$, where $A$ represents the area $66.5 \times 66.5 \mathrm{~mm}^{2}$ and $M=1024 \times 1024$ the number of grid points in that area. $n_{0}$ is now proportional to $2 \pi r d r$ for sufficiently small $r$, but $n_{0}(r)$ goes to zero at $r$ 


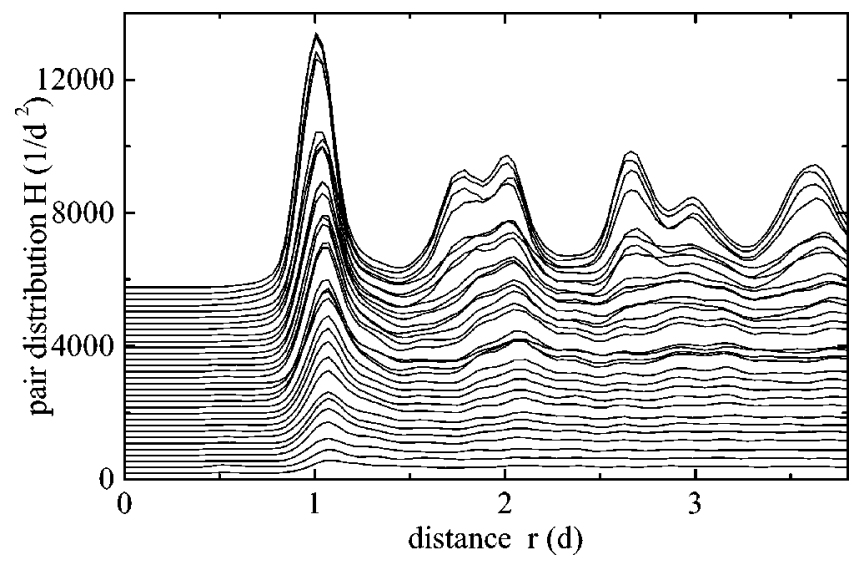

FIG. 4. Pair distribution functions for 32 different filling fractions, obtained at a driving amplitude $A=1 \mathrm{~cm}$, are plotted on top of each other. The lower (upper) line corresponds to $\nu=0.049$ (0.432).

$=66.5 \sqrt{2} \mathrm{~mm}$ due to the finite size of the observation area. Because of the finite grid used for the calculation, the curve is not smooth, however.

We normalize the pair distribution function by $H(r)$ $=2 h(r) / n_{0}(r)$. Note that $n_{0}(r)$ replaces the division by $2 \pi r$, which would be appropriate for an infinite observation window. The normalization procedure gives almost identical results for small values of $r$, but corrects the values obtained for larger distances consistently. In addition, it corrects for the nonsmooth distribution of distances caused by the integer values of coordinates mentioned above. The factor 2 in the normalization formula ensures that the integral over the distribution function $\int H(r) n_{0}(r) d r=N^{2}-N$ reflects the number of particles in the image $N$, while $\int H(r) 2 \pi r d r=N_{c}^{2}$ $-N_{c}$ gives an estimate of the number of pairs that would have been observed without the finite-size effect of the image.

The results for a driving amplitude of $1 \mathrm{~cm}$ are shown in Fig. 4. The pair distribution functions $H(r)$ obtained for different filling fractions are plotted on top of each other. The lowest line corresponds to $\nu=0.049$ (1960 particles), and $\nu$ increases stepwise by 0.01225 , i.e., we show only every sec-

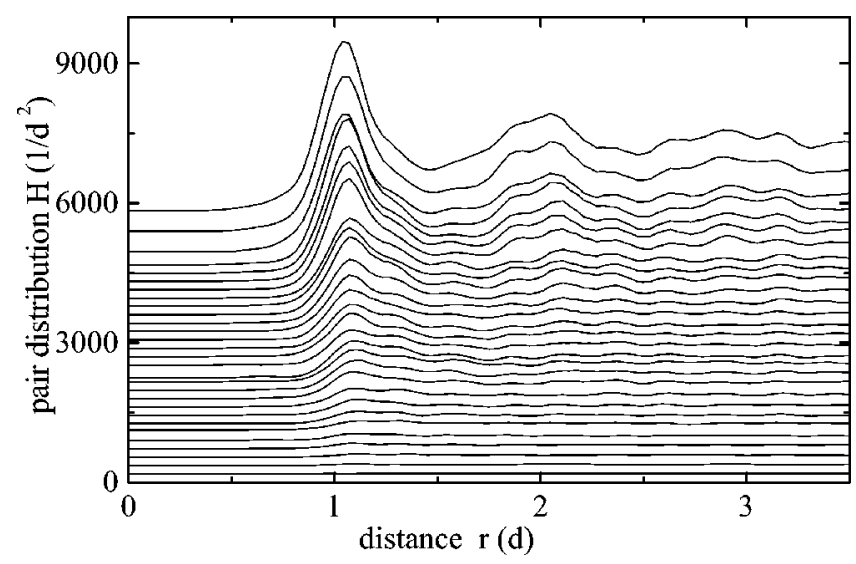

FIG. 5. Pair distribution functions obtained at a driving amplitude $A=2 \mathrm{~cm}$, are plotted on top of each other. An increase in $\nu$ by 0.02 corresponds to an offset of 180. Larger gaps between lines thus represent larger steps in $\nu$.

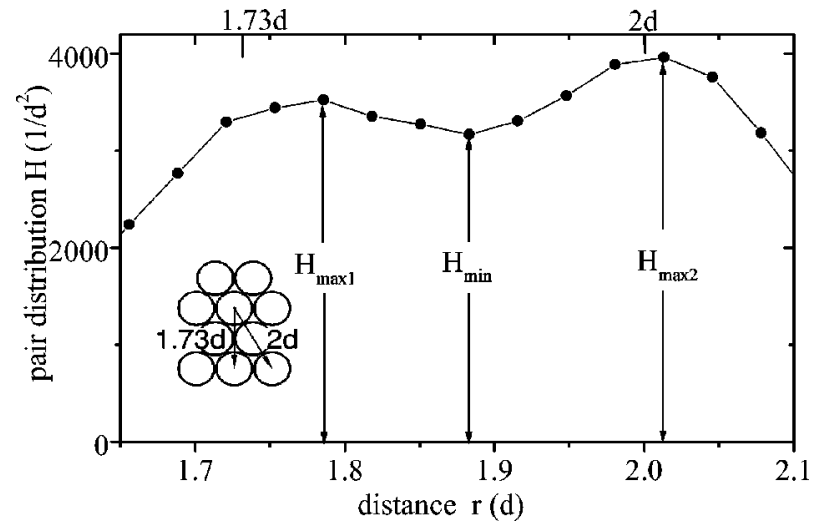

FIG. 6. A part of the pair distribution function obtained at $\nu$ $=0.432$. The arrows indicate the values needed for estimating the dip. The inset illustrates the corresponding distances in a hexagonal array.

ond measurement. The lines are shifted in the vertical direction for clarity, with $\nu=0.01225$ corresponding to an offset of 180 on the $y$ axis. The fact that $H(r)$ is not exactly zero for $r<1$ can be attributed to the final resolution of the camera. Note that this function is not scaled to 1 , the integral $\int_{r=0}^{r=3.7} H(r) 2 \pi r d r$ rather represents the number of distances $r_{i j}$ with $r_{i j}<3.7 d$ found between pairs of particles in the corresponding image, taking into account its finite size. The increase of this integral for higher-filling fractions is clearly observable. In addition, local maxima and minima become apparent for increasing filling fractions.

For the larger driving amplitude of $2 \mathrm{~cm}$ these local extrema are less pronounced as demonstrated in Fig. 5. These pair distribution functions resemble those of fluids or amorphous solids.

The most interesting feature of Fig. 4 is the structure of the peak around $2 d$ : It shows a dip for sufficiently large filling fractions. This is illustrated in detail in Fig. 6. As indicated in the inset, the peaks at $2 d$ and $1.73 d$ can be expected in a dense hexagonal packing. The dip between these values is due to the fact that no corresponding distance

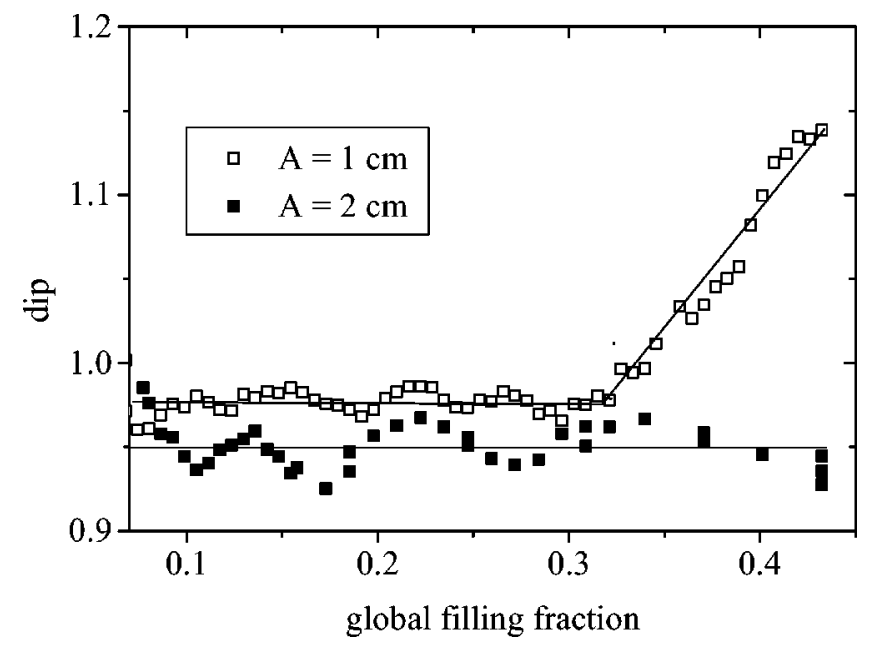

FIG. 7. The dip obtained from $H(r)$ is shown as a function of $\nu$. Open (solid) squares correspond to a driving amplitude of $1 \mathrm{~cm}(2$ $\mathrm{cm})$ at $4 \mathrm{~Hz}$. The lines represent least-squares fits and serve to determine the phase transition. 
can be found in that packing. Thus, the existence of this dip, which is not expected for a gaslike or fluidlike granulate, can be considered as a characteristic feature of a crystallike packing.

We quantify the dip by $0.5\left(H_{\max 1}+H_{\max 2}\right) / H_{\min }$ and plot this value for different filling fractions in Fig. 7. The change in the slope at a global filling fraction of about 0.3 for the driving amplitude of $1 \mathrm{~cm}$ (open squares) clearly indicates the granular phase transition from fluidlike to crystallike behavior.

In contrast, no transition can be detected at the larger driving amplitude of $2 \mathrm{~cm}$. Adding more spheres to the cluster in this case leads to a three-dimensional arrangement of the particles, rather than to a regular two-dimensional packing.

In conclusion, we have detected a granular phase transition when increasing the filling fraction in a horizontally vibrated container, and described it quantitatively by making use of an order parameter obtained from the pair distribution function. The fact that this transition occurs for moving particles makes the experiment an attractive model for the collective behavior of freely moving particles like sand in an hourglass or cosmic dust. Because this phase transition is observed in a two-dimensional system it should be particularly suitable for a theoretical analysis in terms of a molecular-dynamics simulation. From an experimental point of view it is tempting to simplify the geometry even further by replacing the spheres with disks, or by using different kinds of "heating," like circular or elliptic oscillations instead of linear ones. It remains to be seen if the phase transition persists.

We thank Sébastien Aumaître, Jürgen Bläsing, Vidar Frette, and Christof Krülle for helpful hints and clarifying discussions. We are grateful for support from the Deutsche Forschungsgemeinschaft under Contract No. Re 588/12.
[1] H.M. Jaeger, S.R. Nagel, and R.P. Behringer, Rev. Mod. Phys. 68, 1259 (1996).

[2] I. Goldhirsch and G. Zanetti, Phys. Rev. Lett. 70, 1619 (1993).

[3] J.S. Olafsen and J.S. Urbach, Phys. Rev. Lett. 81, 4369 (1998).

[4] W. Losert, D.G.W. Cooper, and J.P. Gollub, Phys. Rev. E 59, 5855 (1999).

[5] G.H. Ristow, G. Straßburger, and I. Rehberg, Phys. Rev. Lett. 79, 833 (1997).

[6] A. Kudrolli, M. Wolpert, and J.P. Gollub, Phys. Rev. Lett. 78,
1383 (1997).

[7] S.E. Esipov and T. Pöschel, J. Stat. Phys. 86, 1385 (1997).

[8] G. Straßburger, A. Betat, M.A. Scherer, and I. Rehberg, in Traffic and Granular Flow, edited by D.E. Wolf and A. Bachem (World Scientific, Singapore, 1996).

[9] A. Betat, C.M. Dury, I. Rehberg, G.H. Ristow, M.A. Scherer, and G. Straßburger, in Evolution of Spontaneous Structures in Dissipative Continuous Systems, edited by F.H. Busse and S.C. Müller (Springer-Verlag, Berlin, 1998). 